\title{
Infrared spectroscopy on the nanometer-scale
}

\author{
T. Taubner*, R. Hillenbrand* and F. Keilmann* \\ *Max-Planck-Institut für Biochemie, Am Klopferspitz 18a, D-82152 Martinsried, Germany, \\ taubner@biochem.mpg.de
}

According to classical optics the spatial resolution that can be obtained in microscopy is limited by diffraction, to about half the illumination wavelength $\lambda$. Scanning near-field microscopy (SNOM) defeats this limitation by exploiting the evanescent field propagated through a subwavelength aperture of a tapered fiber tip, yielding a spatial resolution approximately equal to the aperture diameter. However, the resolution achievable remains limited by power loss as the aperture becomes smaller than $\lambda / 10$. This is a severe restriction, especially when doing microscopy with mid-infrared wavelengths.

Scattering-type SNOM (s-SNOM) uses the optical near-field interaction between an illuminated metal or dielectric probe tip and the sample surface[1-5]. Its spatial resolution is not limited by diffraction but rather by the actual size of the scattering probe tip $(<20 \mathrm{~nm})$. We show that it is possible to distinguish between material classes at a resolution of approx. $30 \mathrm{~nm}$ already in a singlewavelength experiment (Figure 1). We image a three-component test-sample (Au, Si ,PS) at two widely separated wavelengths. Our results are evidence that the imaging process of s-SNOM is wavelength-independent, namely that the resolution is mainly determined by the tip's properties, and that the contrast is given by the refractive index of the sample. This categorizes s-SNOM contrast into the material classes of metals, semiconductors, and polymers enabling a simple, high resolution material-specific mapping of nanosystems $[6,7]$.

The full advantage of the infrared illumination is the sensitivity to molecular or crystal vibrations. Performing spectroscopy at frequencies of these (molecular or lattice) oscillators goes far beyond the material contrast, because structural information can be obtained by looking at the spectral behaviour of these oscillators. We show that a near-field spectrum can indeed differ strongly from a spectrum obtained in a far-field absorbance experiment. As a model system for a strong oscillator, we investigate a polar sample in a frequency range where lattice vibrations (phonons) are excited and find the near-field interaction strongly enhanced.

Imaging a partly gold-covered $\mathrm{SiC}$ surface in our s-SNOM at various wavelengths in the midinfrared, we observe a strongly increased signal on $\mathrm{SiC}$ at $920 \mathrm{~cm}^{-1}$, exceeding the signal on gold more than 20 times[5]. By changing frequency of the illuminating light and comparing the signals obtained on $\mathrm{SiC}$ with the signals on gold we obtain a sharply resonant spectrum (Figure 2). This spectral response is completely different from the far-field reststrahlen band of $\mathrm{SiC}$, which shows a much broader signature. Comparing theory (solid line, scaled to $60 \%$ ) with experiment, we find a good agreement of the strong resonant behaviour in both, resulting in an amplitude contrast that ranges over almost 3 orders of magnitude.

This new, phonon-enhanced near-field interaction allows to study phonon-resonant nanosystems (analogous to plasmon-resonant metal particles). It can also be used to image local chemical and 
crystal structure of nanoscale polar materials, e.g. to investigate the process of biomineralization in bone or teeth.

\section{References}

[1] F. Zenhausern et al., Science, 269, 1083 (1995).

[2] A. Lahrech et al., Optics Letters, 21, 1315 (1996).

[3] B. Knoll and F. Keilmann, Nature, 399, 134 (1998).

[4] B. Knoll and F. Keilmann, Applied Physics Letters, 77, 3980 (2000).

[5] R. Hillenbrand et al., Nature, 418, 159 (2002)

[6] R. Hillenbrand and F. Keilmann, Applied Physics Letters, 80, 25 (2002).

[7] T. Taubner et al., Journal of Microscopy, in press(2003)

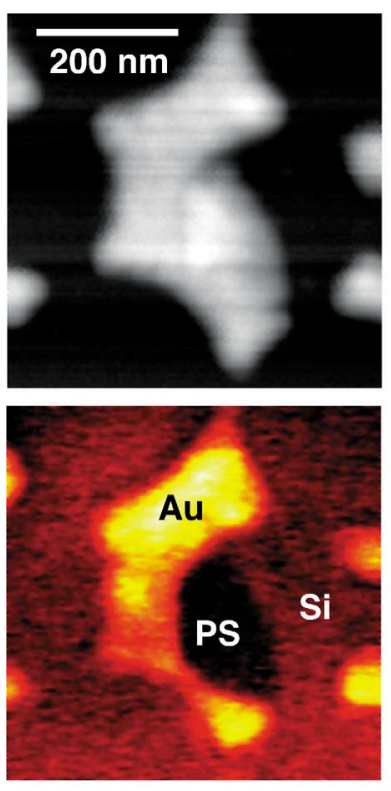

$\lambda=633 \mathrm{~nm}$
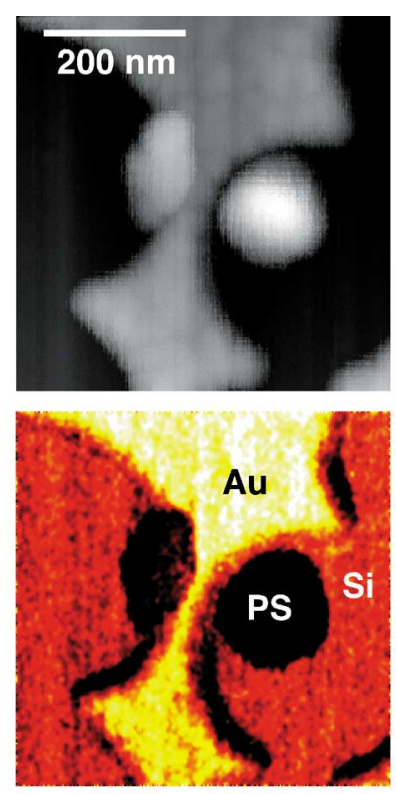

$$
\lambda=9.7 \mu \mathrm{m}
$$

FIG. 1. Topography (grey, top) of a three-component test sample. Optical images (bottom) with visible (left) and infrared (right) illumination.
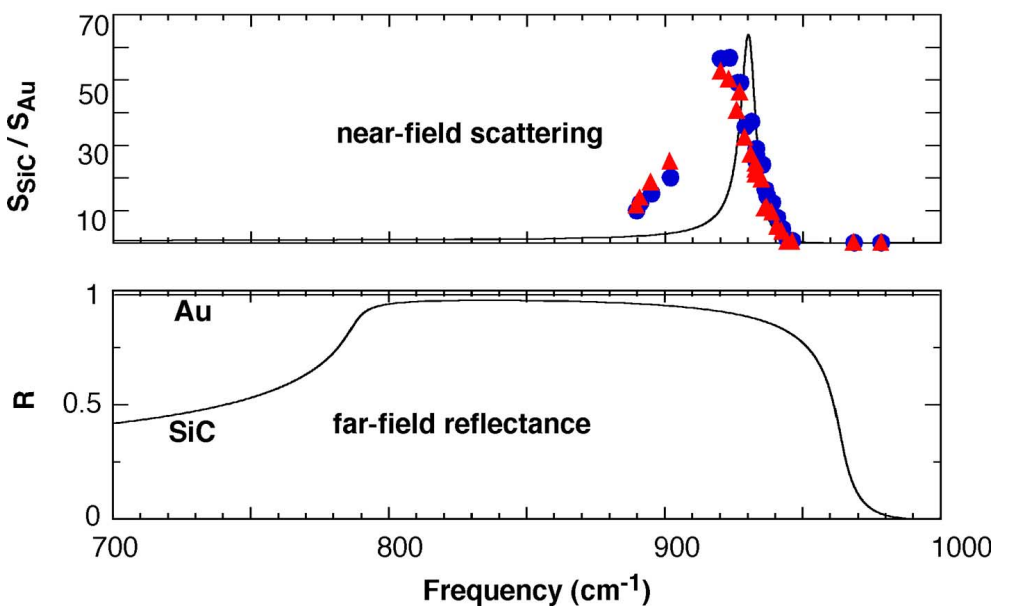

FIG. 2. Comparison of far-field reflection (bottom) with near-field scattering amplitude spectra of $\mathrm{SiC}$ (top), revealing the new phenomenon of polaritonic near-field resonance 